\title{
Customers or research participants?: Guidance for research practices in commercialization of personal genomics
}

\author{
Sara L. Tobin, PhD¹, Mildred K. Cho, PhD¹, Sandra S.-J. Lee, PhD¹, David C. Magnus, PhD', \\ Megan Allyse, PhD'1, Kelly E. Ormond, MS, $\mathrm{CGC}^{1,2}$ and Nanibaa' A. Garrison, PhD ${ }^{1}$
}

Applications of genetic and genomic technologies underlie several emerging businesses offering assessments of individual variations in DNA directly to consumers. Yet customers of these companies often become transformed into research participants, sometimes as a condition of purchase. The subtle nature of this transition has implications both for consumers and for the research enterprise in general. To assess how companies are negotiating this transition, we examined posted research policies from four companies regarding the ethical principle of autonomy (i.e., informed and voluntary consent) that is key to research involving human subjects. In such novel situations, professional expertise may be helpful to guide applications of ethical principles for protection of human subjects.

This commentary suggests that sound human subjects practices may ultimately contribute both to a robust consumer market and to public understanding of the research enterprise. We are in the midst of unprecedented advances in genetic and genomic technologies. Current microchips can query over two million genomic single-nucleotide variations simultaneously. Such techniques are vital to novel commercial approaches in which personal genomics companies sell genomic assessments direct-to-consumers (DTC). Most DTC companies currently analyze the single-nucleotide variations in an individual customer's sample and correlate these small sequence variations with characteristics identified through published human subjects research studies. Such studies identify statistical associations between defined single-nucleotide variations and the probability of characteristics and disease conditions. Collections of these statistical associations are communicated to purchasers as personal genome profiles.

In this commercial context, potential customers contact the company to consider purchasing information about their own genetic predispositions and/or ancestry, and by extension, those of their families. The information sought may be about correlation with "recreational" characteristics, ${ }^{1}$ such as the ability to taste or smell certain substances, but it has equal potential to be medical in nature, such as, for example, reporting a substantially elevated risk of cancer. Regardless of the type of genomic data, the primary goal of these customers is to purchase information about their personal genetic makeup, a context that is very different from that of a volunteer who considers participation in a research biobank or a specific research project.

After the purchased analysis has been sent to the customer, companies often use customer samples and data internally for purposes such as quality control. Such internal uses are not generally categorized as research involving human subjects. However, human subjects concerns do become relevant for research studies that are designed to add to general knowledge and usually lead to publications. To date, only one DTC company has based academic publications on data from the genomes of its customers ${ }^{2,3}$, so the eventual level of interest in research publication by personal genomics companies is difficult to predict. However, because the DTC genetic testing industry is still young, we believe that it is important to explore the implications of research on customer samples.

It is possible that personal genomics companies have not anticipated the challenging ethical and regulatory issues involved in using samples and data from their customers for research. Interpretation of federal human subjects regulations requires expertise, and the regulations may or may not apply to non-federally funded research on commercially derived samples. Whether or not federal regulations apply, we argue that it is in the best interests of DTC companies, their customers, and the future of personalized medicine to address potential issues in research ethics early on. Ideally, companies will make good-faith efforts to achieve federal standards for human subjects protections regardless of whether the company is legally required to do so.

Three companies have led the way in the commercial genomic profiling market: deCODE, Navigenics, and 23andMe, and Pathway Genomics has recently emerged as well. All four

${ }^{1}$ Center for Biomedical Ethics, School of Medicine, Stanford University, Stanford, California, USA; ${ }^{2}$ Department of Genetics, School of Medicine, Stanford University, Stanford, California, USA. Correspondence: Sara L. Tobin (tobinsl@stanford.edu) 
companies have posted detailed policies for consumers on their respective company websites. The posted policies describe how customer samples and data will be handled. Ideally, this information will foster the ability of consumers to make informed and voluntary choices about research participation. In this commentary, we emphasize autonomy as a central issue, but we acknowledge that other important issues remain, such as measures to ensure effective informed consent, confidentiality, ability to withdraw samples and data, and returning research results. As we describe policies that influence the ability of research participants to make informed and voluntary choices, our purpose is to point out that even with the best efforts to implement existing research regulations, it is useful to anchor such efforts in key ethical principles when faced with novel situations, such as new commercial uses for technological innovations. Selected aspects of the policies posted by the four companies are presented in Table $\mathbf{1}$ and summarized briefly in the following.

Substantial variability in policies related to autonomy may be seen in Table 1. For example, deCODE uses completely separate agreements for commercial services and for research. Thus the company pledges to request specific consent for research use of customer samples. This practice enhances autonomy by drawing the customer's attention to the change in the use of their samples and data. Pathway Genomics intends to "develop new genetic reports and/or services, but not publish the results in scientific literature." This policy thus permits internal improvements and quality control, but does not raise research concerns.

Navigenics posts a document in which the consumer authorizes the DNA analysis and also grants permission to use the customer's sequence and phenotypic information for "internal research." However, the description includes research to "discover or validate associations between certain variations and health conditions or traits, as well as other insights regarding human health," with the intention "to publish our findings and insights ... to further the advancement of scientific and medical research, with the goal of improving health care." Thus Navigenics customers cannot avoid authorizing the use of their genetic data for research. We feel that this policy erodes the autonomy of individuals purchasing their genome profiling services.

With 23andMe (https://www.23andme.com), it was possible to compare posted documentation both before and after revisions were incorporated following the company's consultation with an institutional review board (IRB). The consultation was prompted when researchers at 23andMe used customer samples and data for genomic research intended for publication without a research ethics review. However, a review was required by the editors of the journal to which the publication was submitted, ${ }^{4}$ permitting a comparison of documentation posted in April 2010 with the revised versions posted on 24 June 2010.

The April 2010 version was titled "Consent and Legal Agreement," and customers who purchased genotyping were required to contribute their genetic information to 23andMe's research efforts as a condition of sale. Customers were also required to waive property rights in potential discoveries and to agree to hold the company harmless for possible damages. Such requirements diminish the autonomy of research participants. However, following the IRB review, research participation is now specifically described as voluntary, and all customers are asked to participate by granting researchers permission to access their information. The new versions of the consent and privacy documents enhance autonomy of participants by separating research information from both commercial legal information and disclosures designed to protect the company.

These examples illustrate the challenges encountered by companies seeking to develop commercial products and services in the era of personalized medicine. As genomic research moves into the commercial realm, new companies may be focused primarily on survival or may simply view customer samples as

\section{Table 1 Research use of customer samples and data}

\begin{tabular}{|c|c|c|c|c|c|}
\hline & DeCode ${ }^{a}$ & Pathwayb & Navigenics ${ }^{c}$ & $\begin{array}{l}\text { 23andMe }{ }^{d} \text { before } \\
\text { IRB consult }\end{array}$ & $\begin{array}{l}\text { 23andMe } e^{e, f} \text { after } \\
\text { IRB consult }\end{array}$ \\
\hline Customers who purchase genotyping must also consent to research & No & No & Yes & Yes & No \\
\hline Customer samples are used for internal research (e.g., quality control) & $\mathrm{N} / \mathrm{A}$ & Yes & Yes & Yes & Yes \\
\hline Customer samples are used for research for general knowledge & N/A & No & Yes & Yes & Yes \\
\hline $\begin{array}{l}\text { Customers are required to sign legal waivers covering research } \\
\text { participation }\end{array}$ & $\mathrm{N} / \mathrm{A}$ & N/A & Yes & Yes & No \\
\hline
\end{tabular}

CO, human subjects review is required for outside collaborators only; IRB, institutional review board; N/A, not applicable because company does not do research on customer samples; ND, not described on website.

Information drawn from company websites:

adeCODEme Genetic Scan Service Agreement and Informed Consent, 2010. http://www.decodeme.com/service-agreement. Accessed 2 August 2010.

bPathway Genomics Privacy Policy, 2012. https://www.pathway.com/about-us/privacy-policy. Accessed 28 April 2012.

'Navigenics Informed Consent, Health Compass, 2010. http://www.navigenics.com/visitor/what_we_offer/our_policies/informed_consent/health_compass/. Accessed

2 August 2010

23andMe. Claim Your Kit. Consent and Legal Agreement, 2010. No longer posted in original form. Version 1.3 (identical to original with additional section). https:// www.23andme.com/about/consent/?version=1.3. Accessed 3 August 2010.

e23andMe. Consent Document, 2010. https://www.23andme.com/about/consent/. Accessed 3 August 2010

${ }_{2} 3$ andMe. Privacy Statement, 2010. https://www.23andme.com/about/privacy/. Accessed 2 August 2010; 23andMe. Summary of Changes to the 23andMe Privacy

Statement, 2010. No longer posted. Accessed 27 June 2010. 
a convenient resource. If a DTC company plans to use samples for research, the ethical principles that serve as the basis of human subjects protections should be respected. These include measures to ensure autonomy, such as voluntary informed consent for the use of samples for research purposes.

Based on extensive experience with research ethics consultation ${ }^{5}$ and on the helpful modifications to 23andMe's disclosures to consumers following their consultation with an IRB, we contend that companies can benefit substantially from engaging in IRB review or ethics consultations early in the design of the research initiatives that underlie their business models. Although we are not arguing for a change in regulation, or that journals are the best-equipped or appropriate bodies to provide oversight for the long term, we do feel that it is important for all research entities (including commercial genomics companies) to remain focused on the ethical principles that gave rise to human subjects regulations in the first place. For example, companies should use separate informed consent processes for commercial and research activities, have explicit policies about secondary use of samples (including who has access to samples and data and the types of research and lengths of time during which samples could be used), and obtain IRB review of research. These are early days in commercialization of genetics and genomics assessment companies, and it is appropriate for these companies to take seriously the responsibility of educating their customers, not only about the interpretations of the genotyping data they are purchasing, but also about their dual status as customers and as research participants. The commercial viability of genetic and genomic assessments may depend on how well the companies foster public confidence by building ethical principles into their research programs.

\section{ACKNOWLEDGMENTS}

The authors acknowledge support of a P50 center grant from the National Institutes of Health titled Center for the Integration of Research on Genetics and Ethics (CIRGE), HG003389-06. N.A.G. was supported by postdoctoral National Research Service Award F32 HG005931 from the National Institutes of Health. We thank Maya Wolpert for expert assistance.

\section{DISCLOSURE}

The authors declare no conflict of interest.

\section{REFERENCES}

1. Evans JP. Recreational genomics: what's in it for you? Genet Med 2008;10:709-710.

2. Eriksson N, Macpherson JM, Tung JY, et al. Web-based, participant-driven studies yield novel genetic associations for common traits. PLoS Genet 2010. http://www.plosgenetics.org/article/info\%3Adoi\%2F10.1371\%2Fjournal. pgen. 1000993.

3. Do CB, Tung JY, Dorfman E, et al. Web-based genome-wide association study identifies two novel loci and a substantial genetic component for Parkinson's disease. PLoS Genet 2011. http://www.plosgenetics.org/article/ info\%3Adoi\%2F10.1371\%2Fjournal.pgen. 1002141.

4. Gibson G, Copenhaver GP. Consent and internet-enabled human genomics. PLOS Genet 2010. http://www.plosgenetics.org/article/ info\%3Adoi\%2F10.1371\%2Fjournal.pgen. 1000965.

5. Cho MK, Tobin SL, Greely HT, McCormick J, Boyce A, Magnus D. Strangers at the bedside: research ethics consultation. Am J Bioethics 2008;8:4-13. 